\title{
Spectral Modeling of an Oscillating Surge Wave Energy Converter with Control Surfaces
}

\author{
Nathan Tom ${ }^{\mathrm{a}, *}$, Michael Lawson ${ }^{\mathrm{a}}$, Yi-Hsiang Yu ${ }^{\mathrm{a}}$, Alan Wright ${ }^{\mathrm{a}}$ \\ ${ }^{a}$ National Renewable Energy Laboratory, MS 3811, 15013 Denver West Parkway, Golden, CO, 80401
}

\begin{abstract}
The aim of this research is to use spectral techniques in evaluating the irregular wave performance of a novel wave energy converter concept that combines an oscillating surge wave energy converter with active control surfaces. The control surfaces allow the wave energy converter to have a time-varying geometry that enables the hydrodynamic exciting and radiation coefficients to be altered. In the current state of development the device geometry is controlled on a sea-to-sea time scale and combined with control of the power take-off (PTO) on a wave-to-wave time scale to maximize power capture, increase capacity factor, and reduce design loads. Analysis begins with the application of linear hydrodynamic theory to evaluate the device performance in terms of absorbed power, foundation loads, and accumulation of fatigue damage on the PTO. To determine the linear PTO damping coefficient that maximizes the time-averaged absorbed power for a given sea state, an optimization problem was constructed while incorporating a motion constraint on the maximum pitch amplitude of motion. The inclusion of the motion constraint prevents linear scaling of the performance results with the significant wave height. Previous studies on the modeling of oscillating surge wave energy converter designs have included the consideration of nonlinear hydrodynamics. Therefore, a quadratic viscous drag moment was added to the system dynamics through use of a quasi-linear viscous damping coefficient. The same performance quantities were calculated for both the linear and nonlinear models while assuming an irregular wave surface elevation described by a Bretscheider spectrum. One major effect of including the viscous drag moment was flattening of the capture width and structural load curves with respect to the wave spectrum peak frequency while reducing the sensitivity with respect to the significant wave height compared to the linear analysis.
\end{abstract}

\section{(C) 2016 Published by Elsevier Ltd.}

Keywords: oscillating surge wave energy converter, variable structures, irregular wave, nonlinear optimization, viscous linearization, fatigue damage

\section{Introduction}

The worldwide marine renewable energy resource has the potential to significantly reduce the world's consumption of fossil fuels. The still nascent wave energy industry hosts a wide diversity of technologies, yet no convergence toward an optimal design and operation has emerged [1]. The current generation of wave energy converters (WECs) is facing several challenges: 1) the energy capture efficiency is lower than the theoretical maximum, 2) large structures are needed to withstand the hydrodynamic loads, and 3) the price of energy is too high and costs must be driven down

\footnotetext{
${ }^{*}$ Corresponding author

Email addresses: nathan.tom@nrel.gov (Nathan Tom ), Michael.Lawson@nrel.gov (Michael Lawson), Yi-Hsiang.Yu@nrel.gov (Yi-Hsiang Yu), Alan.Wright@nrel.gov (Alan Wright)
} 
before WECs can become commercially viable [2]. Therefore, the success of future WEC technologies will require the development of advanced control methods and structures that actively tune device performance to maximize energy generation under operational conditions and shed hydrodynamic loads in extreme sea states to reduce the structural mass and overall cost [3].

To address these issues the National Renewable Energy Laboratory has been developing a novel WEC concept that combines an oscillating surge wave energy converter (OSWEC) with active control surfaces [4]. The concept of controllable airfoils applied to wave energy conversion was pioneered by Atargis Energy Corporation with its cycloidal device [5]. The concept of large-scale geometric changes has been considered in the design of Weptos [6], though the focus has been on its survival mode. The concept developed in this article is more similar to a pitching device with a rotatable flap [7]; however, this work increases the number of rotatable surfaces for greater refinement of the hydrodynamic properties. The proposed design is expected to assist in tuning the hydrodynamic properties to match those of the incident wave climate while shedding loads in larger seas to increase its operational range.

The development of nearshore oscillating surge wave energy devices in recent years has been led by Aquamarine Power's Oyster [8], AW-Energy Oy's Waveroller [9], and Resolute Marine Energy's Surge WEC [10]. In addition, Langlee Wave Power [11] is currently developing a floating dual-flap OSWEC for deepwater deployment; however, these designs consist of a fixed geometrical body that generally does not operate as a resonant device [12] but relies on control of the power-take-off (PTO) system to optimize power capture. Point absorbers are generally designed to be resonant devices, though their motion is generally narrow-banded with high extraction efficiencies around a small frequency range near the natural period of the device. References [7, 13] investigated the ability to tune the natural period of a pitching WEC by altering the mass moment of inertia by shifting the internal mass distribution. A device with a controllable geometry would not only be able to shed loads, but also tune the hydrodynamic coefficients to match the natural period of the device with the peak excitation period of the sea spectrum to broaden its effective operating range. Increasing the number of control surfaces will allow for greater tuning of the hydrodynamic excitation and radiation coefficients and will reduce the complexity that comes with pumping ballast water [7].

This article begins with an introduction to the device concept and discussion of the sensitivity in hydrodynamic properties with respect to various device geometries chosen for analysis. Next, modeling of the OSWEC in regular waves is reviewed to provide the preliminaries for extension into spectral models [14, 15]. The performance quantities of interest include the rotational amplitude of motion, the time-averaged absorbed power from the PTO system, and the foundation loads required to keep the device fixed to the sea floor. The theory behind the spectral modeling of irregular waves is then introduced to obtain short-term performance statistics. OSWEC designs require considerations for modeling nonlinear hydrodynamics $[12,16]$; thus, a nonlinear quadratic viscous drag moment will be added to the system dynamics. To maximize the absorbed power for a given sea state, a nonlinear optimization problem was constructed to solve for the optimal linear PTO damping coefficient while satisfying a given motion constraint. Because the optimization focused solely on power generation, the corresponding foundation loads and fatigue damage accumulation to the PTO were calculated to highlight the benefit of controlled surfaces to shed loads in greater sea states to increase the lifetime of the device.

\section{Device Description and Hydrodynamic Modeling}

A preliminary analysis of the device concept's feasibility was performed in [17], which showed that an actuated geometry could increase the capacity factor while maintaining a baseline power performance level. The concept was further developed in [4], which studied how the absorbed power and hydrodynamic loads varied with the device width and thickness. As described in the previous studies, the main body of the OSWEC has been replaced with a set of identical flaps that may rotated, see Fig. 1. The flaps will be allowed to pitch about their center of rotation with the flap pitch angle, $\varphi$, measured positive clockwise from the radial axis of the body, see Fig. 2 . The flaps were modeled as ellipses to provide a streamlined shape when the flaps are rotated parallel to the direction of wave propagation, $\varphi=\pi / 2$, in an attempt to minimize viscous loses. The geometric dimensions used in this study can be found in Table 1. The structural mass will be evenly distributed; as such, the pitch mass moment of inertia, $I_{55}$ and the linear restoring moment coefficient, $C_{55}$, will remain constant. For this study the structural mass density, $\rho_{m}$, was set to half the fluid density, $\rho$.

For this study hydrodynamic coefficients were obtained from WAMIT version 7.0 [18] at a spacing of 0.01 $\mathrm{rad} / \mathrm{s}$ for wave frequencies of $0-3.5 \mathrm{rad} / \mathrm{s}$. The flaps were placed in either the fully open or closed configuration 

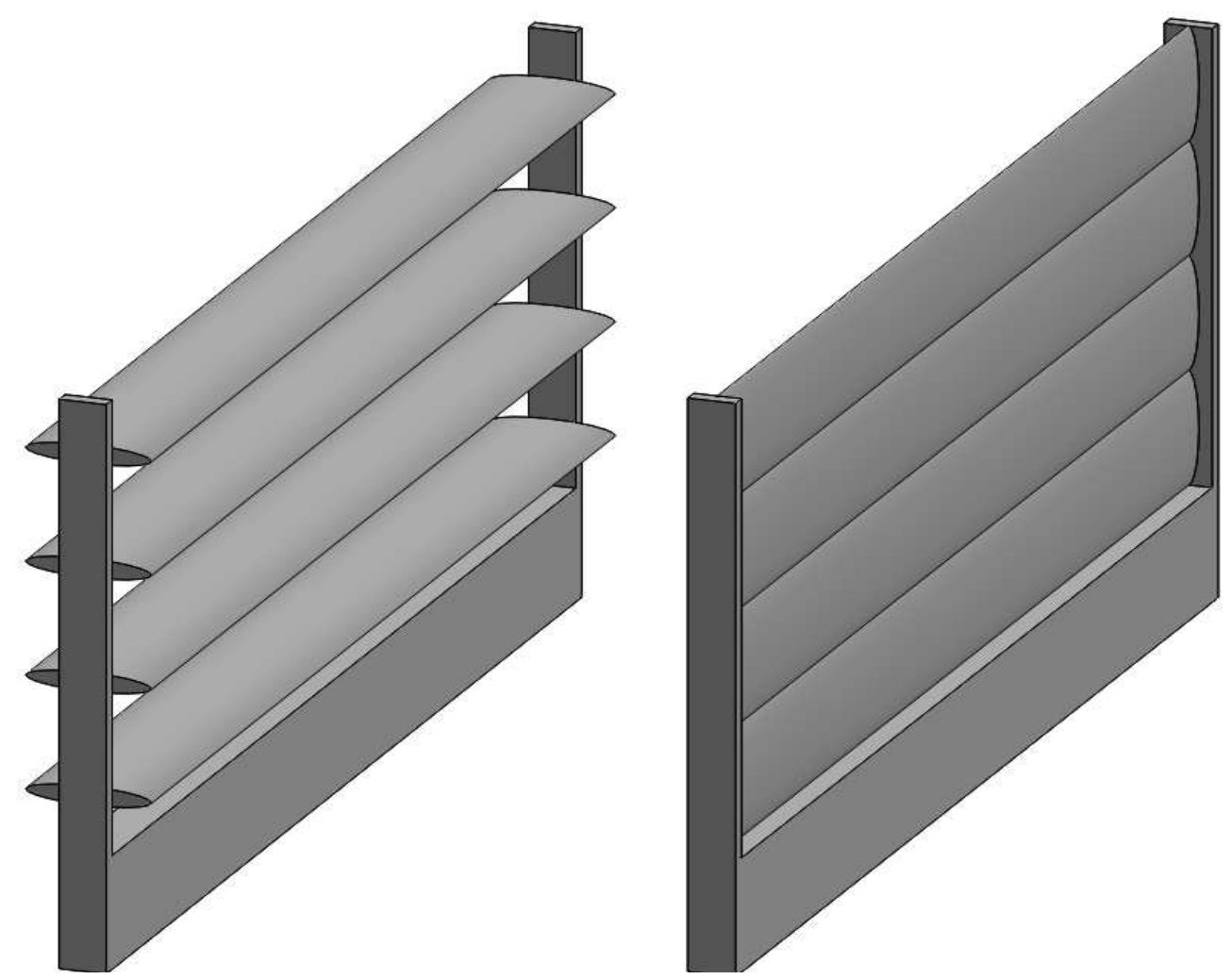

Figure 1. Solidworks rendering of the OSWEC. Left: Perspective view of fully open configuration (4 flaps open) and right: perspective view of fully closed configuration (0 flaps open).

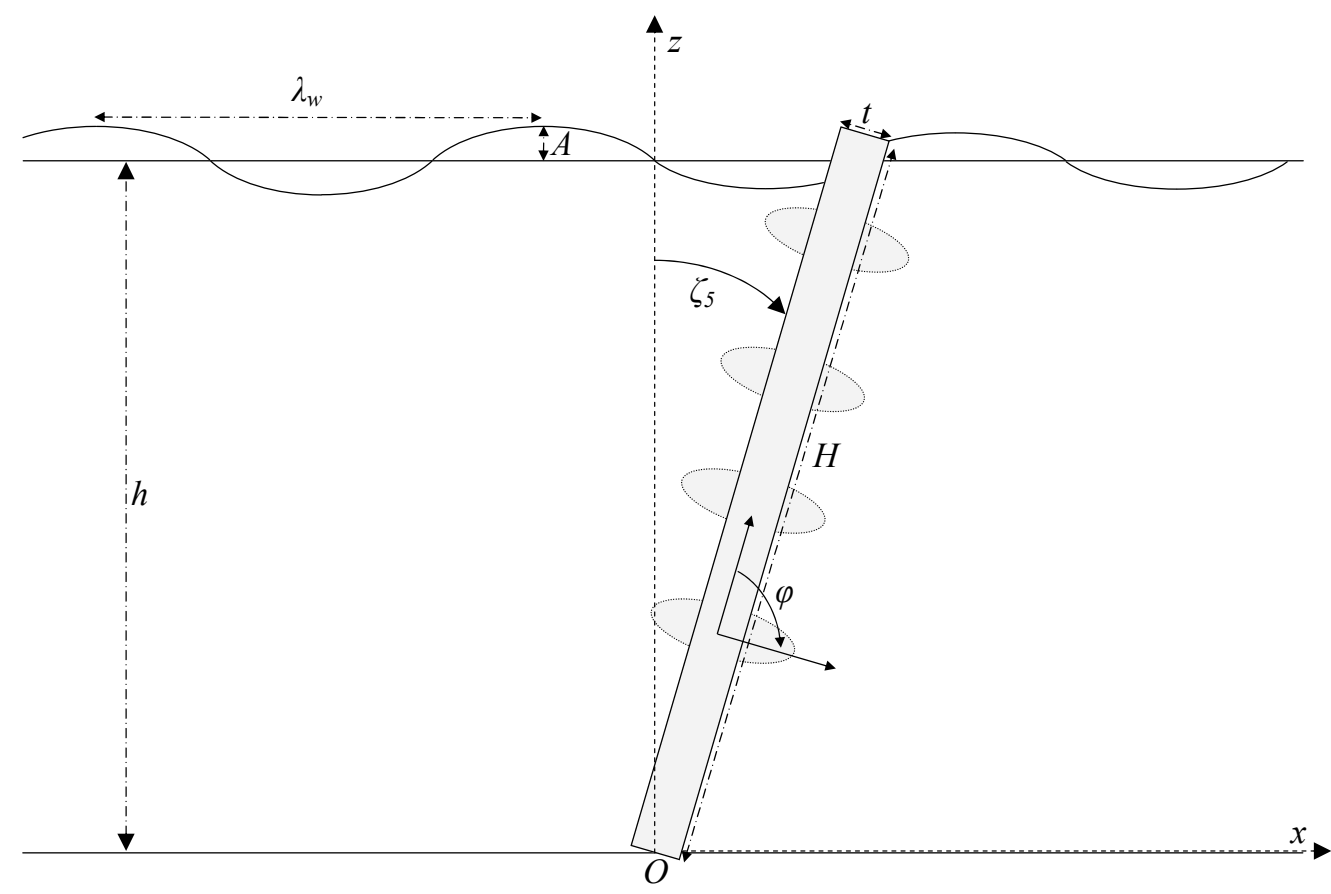

Figure 2. Coordinate system for the device under investigation. 
corresponding to $\varphi=\pi / 2$ and $\varphi=0$, respectively. This work considers four separate geometric configurations that consist of starting with the top flap open, one flap, and opening the next three flaps one at a time in sequential order. Results for the geometric configuration with zero open flaps is not discussed, because it has been previously researched and is not required to demonstrate the benefit of actuated control surfaces. The hydrodynamic coefficients for each geometric configuration considered can be found in Fig. 3. It is evident from Fig. 3 that the hydrodynamic coefficients do not scale linearly, when each additional flap is closed, with the largest increase corresponding to the closure of the second flap from the top of the device. As each flap is closed, the added moment of inertia increases up to 35 times the mass moment of inertia. Because the mass moment of inertia and hydrostatic restoring moment remain constant, for each geometric configuration, it is evident that the natural period will increase as the number of closed flaps increases.

\section{Regular Wave Analysis}

\subsection{Frequency Domain Equation of Motion}

It is common practice to calculate the response amplitude operator to access the performance of a WEC. For an incident wave described by:

$$
\eta(x, t)=\mathfrak{R}\left\{-\left.\frac{1}{g} \frac{\partial \phi_{I}}{\partial t}\right|_{z=h}\right\}=\mathfrak{R}\left\{A e^{i(\sigma t-k x)}\right\}=A \cos (\sigma t-k x),
$$

where $\eta$ is the wave elevation, $g$ is gravitational acceleration, $\phi_{I}$ is the incident wave potential, $A$ is the wave amplitude, $\sigma$ is the wave angular frequency, and $k$ is the wave number, $i=\sqrt{-1}$ is the imaginary unit, and $\mathfrak{R}$ signifies the real component. The OSWEC will pitch about the origin, $O$, which is fixed to the seabed and the corresponding pitch equation of motion is given by:

$$
\frac{\xi_{5}}{A}=\frac{X_{5}}{\left[C_{55}-\sigma^{2}\left(I_{55}+\mu_{55}\right)\right]+i \sigma\left[\lambda_{55}+B_{g}\right]},
$$

where $\xi_{5}$ is the complex amplitude of pitch motion, $X_{5}$ is the complex pitch wave exciting force per unit wave amplitude, $C_{55}$ is the pitch restoring coefficient, $I_{55}$ is the pitch mass-moment of inertia, $\mu_{55}$ is the pitch-added moment of inertia, $\lambda_{55}$ is the wave radiation damping, and $B_{g}$ denotes the linear PTO damping coefficient required for power extraction.

The OSWEC hydrostatic restoring moment is given by:

$$
m_{h}(t)=-\left(\rho \forall r_{b}-m r_{g}\right) g \sin \zeta_{5}(t),
$$

where $\rho$ is the fluid density, $\forall$ is the WEC displaced volume in calm water, $r_{b}$ is the center of buoyancy, $m$ is the WEC mass, $r_{g}$ is the center of gravity, and $\zeta_{5}$ is the time-varying pitch displacement. Equation (3) can be linearized by assuming small rotational motion and making the following approximation $\sin \zeta_{5} \approx \zeta_{5}$.

Table 1. Geometric parameters for hydrodynamic modeling.

\begin{tabular}{|cccccc|}
\hline Water Depth & $h$ & $10 \mathrm{~m}$ & Flap Minor Axis & $t_{f}$ & $1 / 3 \mathrm{~m}$ \\
\hline OSWEC Height & $H$ & $10 \mathrm{~m}$ & Flap Major Axis & $H_{f}$ & $2 \mathrm{~m}$ \\
\hline OSWEC Thickness & $t$ & $3 / 4 \mathrm{~m}$ & Side Support Height & $H_{s}$ & $10 \mathrm{~m}$ \\
\hline OSWEC Width & $w$ & $20 \mathrm{~m}$ & Side Support Thickness & $t_{s}$ & $3 / 4 \mathrm{~m}$ \\
\hline Flap Width & $w_{f}$ & $19.5 \mathrm{~m}$ & Side Support Width & $w_{s}$ & $1 / 4 \mathrm{~m}$ \\
\hline Moment of Inertia & $I_{55}$ & $923.4 \mathrm{t} . \mathrm{m}^{2}$ & Center of Gravity & $r_{g}$ & $3.97 \mathrm{~m}$ \\
\hline Volume & $\forall$ & $72 \mathrm{~m}^{3}$ & Mass & $m$ & $36 \mathrm{t}$ \\
\hline
\end{tabular}




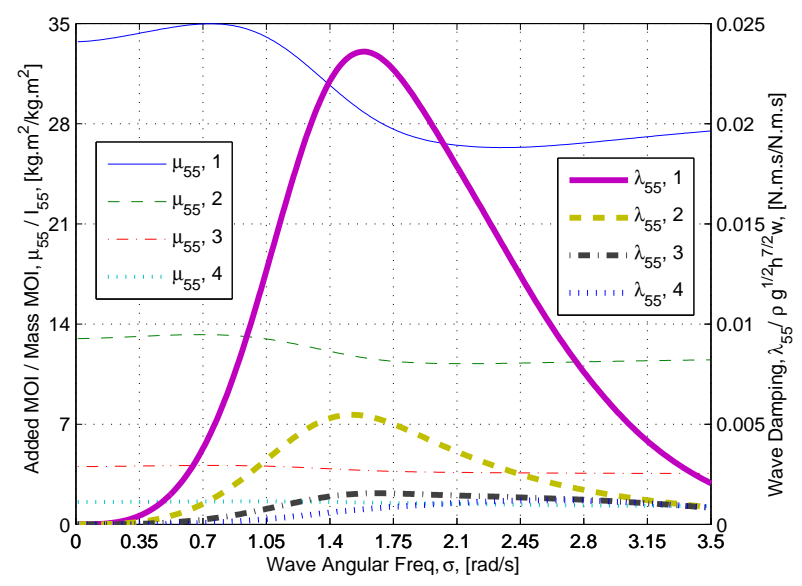

(a) Pitch radiation damping, $\lambda_{55}$, and added moment of inertia, $\mu_{55}$.

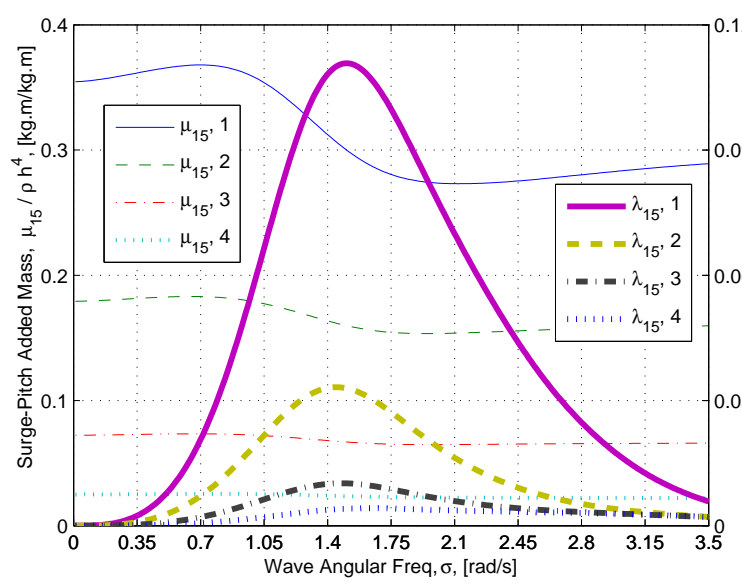

(c) Surge-pitch radiation damping, $\lambda_{15}$, and added mass, $\mu_{15}$.

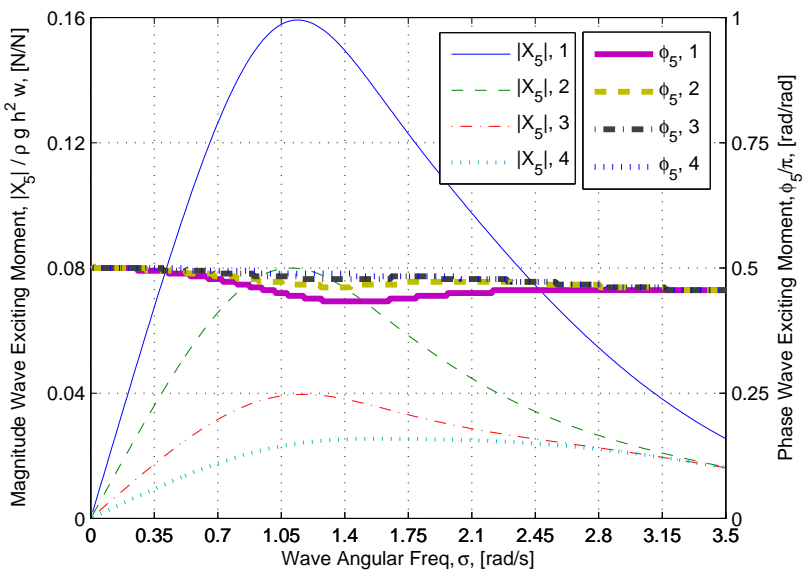

(b) Pitch wave exciting moment magnitude, $\left|X_{5}\right|$, and phase, $\phi_{5}$.

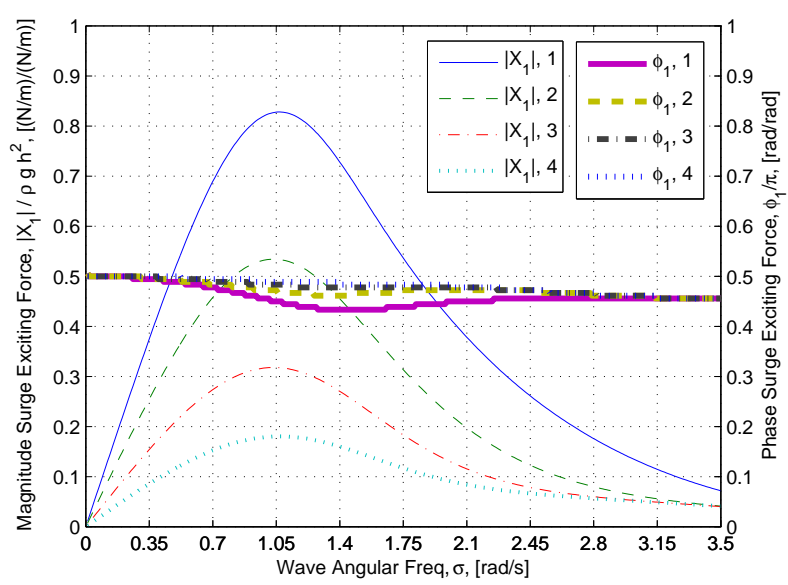

(d) Surge wave exciting moment magnitude, $\left|X_{1}\right|$, and phase, $\phi_{1}$.

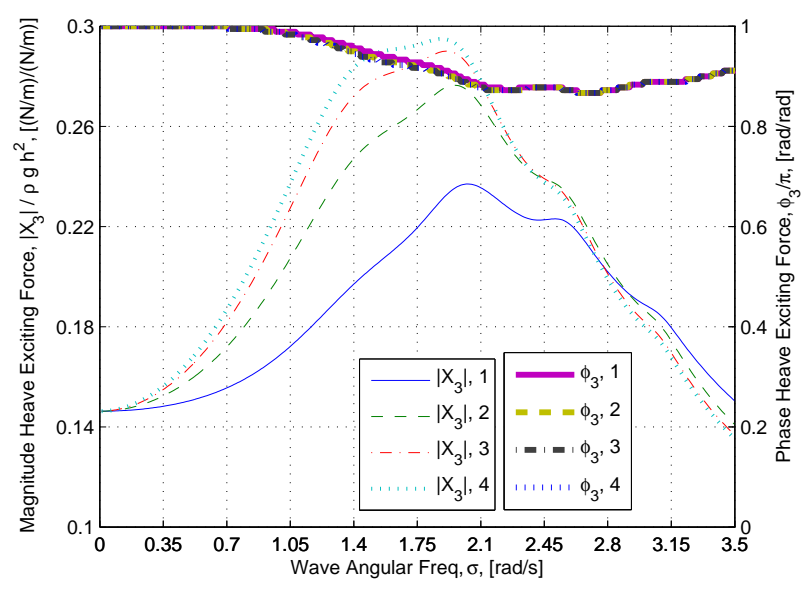

(e) Heave wave exciting moment magnitude, $\left|X_{3}\right|$, and phase, $\phi_{3}$.

Figure 3. Hydrodynamic radiation and wave excitation coefficients for each flap configuration. The numbers in the legend correspond to configurations with 1,2, 3, and 4 open flaps starting from the top. 


\subsection{Power-Take-Off Absorbed Power}

The time-averaged power (TAP) absorbed by the PTO is calculated as follows:

$$
\frac{P_{T A P}}{A^{2}}=\frac{1}{T} \int_{0}^{T} \frac{B_{g}}{A^{2}} \dot{\zeta}_{5}^{2}=B_{g} \sigma^{2}\left|\frac{\xi_{5}}{A}\right|^{2} \sin ^{2}(\sigma t+\Theta) d t=\frac{1}{2} B_{g} \sigma^{2}\left|\frac{\xi_{5}}{A}\right|^{2},
$$

where $T$ is the wave period, $\dot{\zeta}_{5}$ is the pitch velocity, $\Theta$ is the phase (complex argument) of $\xi_{5}$. Equation (2) can be inserted into Eqn. (4) allowing for the optimal PTO damping at each wave frequency to be calculated. The optimal unconstrained absorbed power, for each wave frequency, is then given by:

$$
\left.\frac{P_{T A P}}{A^{2}}\right|_{o p t}=\frac{1}{4} \frac{\left|X_{5}\right|^{2}}{\lambda_{55}} \frac{1}{1+\sqrt{1+\left(\frac{C_{55}-\sigma^{2}\left(I_{55}+\mu_{55}\right)}{\sigma \lambda_{55}}\right)^{2}}},
$$

where at resonance $B_{g}=\lambda_{55}$ leading to the maximum time-averaged absorbed power [19]. The time-averaged power power per unit width, $P_{w}$, contained within a propagating wave can be shown to equal:

$$
P_{w}=\frac{1}{2} \rho g A^{2} V_{g}=\frac{1}{4} \rho g A^{2} \sqrt{\frac{g}{k} \tanh k h}\left[1+\frac{2 k h}{\sinh k h}\right] .
$$

where $V_{g}$ is the wave group velocity and $h$ is the water depth.

\subsection{Foundation Reaction Forces}

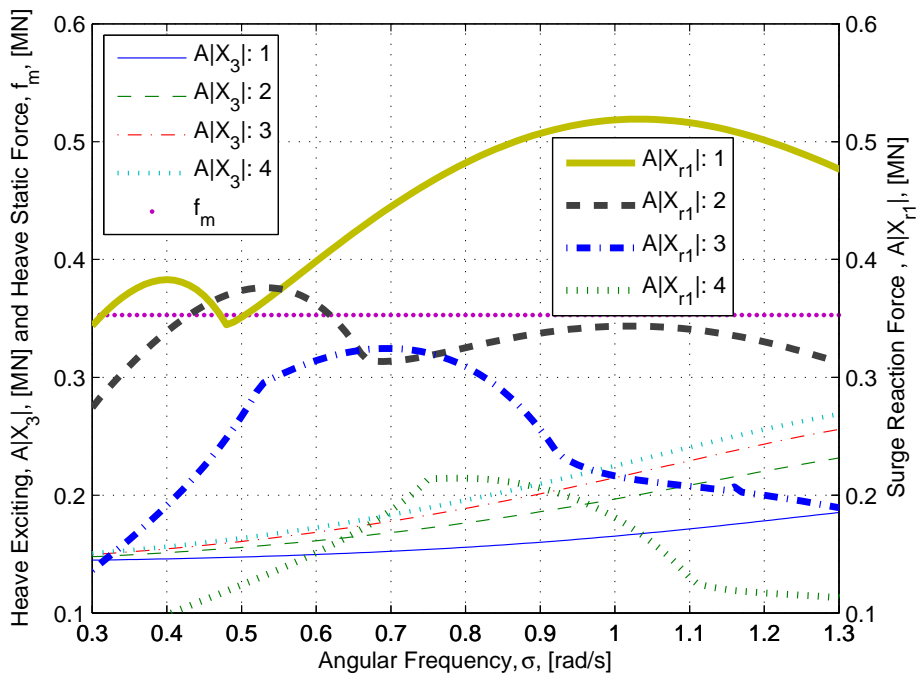

Figure 4. Static versus dynamic foundation reaction force contributions. The values were obtained under regular wave excitation, a wave amplitude of $1 \mathrm{~m}$, with a pitch angular displacement amplitude constraint of $\pi / 6$. The linear PTO damping coefficient was chosen to maximize the timeaveraged absorbed power while staying under the motion constraint, see Eqn. (2) and (4).

The foundation must handle the reaction forces needed to fix the WEC to the seabed. If centrifugal forces are neglected and the body remains symmetric about the vertical plane, the reaction forces in the surge, $X_{r 1}$, and heave, $X_{r 3}$, directions are given by:

$$
\begin{gathered}
A\left(X_{r 1}+X_{1}\right)=\left[-\sigma^{2} \mu_{15}+i \sigma \lambda_{15}\right] \xi_{5} \\
A\left(X_{r 3}+X_{3}\right)=-\underbrace{(\rho \forall-m) g}_{f_{m}},
\end{gathered}
$$


where $X_{1}$ and $X_{3}$ are the complex surge and heave wave exciting force coefficients per unit wave amplitude, $\mu_{15}$ is the surge-pitch added mass, and $\lambda_{15}$ is the surge-pitch wave damping. The static heave reaction force, $f_{m}$, associated with the difference between weight and buoyancy is significant when calculating peak forces, see Figure 4, but it is not a dynamic force and cannot be controlled through either the PTO or changing geometry. The dynamic forces will drive fatigue damage accumulation in the foundation rather than the static mean load. In very small sea states the static heave force will dominate, but as the wave amplitude grows the dynamic component of the reaction forces will have a larger contribution in the magnitude of the reaction force as seen in Figure 4 . The value of $f_{m}$ provided in Figure 4 can be added to the results presented in this work to obtain the total reaction force in the foundation. The dynamic reaction force equations can be simplified and written in terms of the wave exciting force and pitch motion:

$$
\begin{gathered}
X_{r 1}=\left[-\sigma^{2} \mu_{15}+i \sigma \lambda_{15}\right] \frac{\xi_{5}}{A}-X_{1} \\
X_{r 3} \approx-X_{3} .
\end{gathered}
$$

The heave reaction force is not affected by the body motion and varies only with device geometry. The surge foundation reaction force is affected by the OSWEC's pitch motion, which can be controlled by the PTO. The surge foundation force can theoretically be eliminated if the complex pitch amplitude of motion is equal to:

$$
\frac{\xi_{5}}{A}=\frac{X_{1}}{-\sigma^{2} \mu_{15}+i \sigma \lambda_{15}}=\frac{-\mu_{15} \mathfrak{R}\left\{X_{1}\right\}+\frac{\lambda_{15}}{\sigma} \mathfrak{J}\left\{X_{1}\right\}}{\sigma^{2} \mu_{15}^{2}+\lambda_{15}^{2}}+i \frac{-\mu_{15} \mathfrak{J}\left\{X_{1}\right\}-\frac{\lambda_{15} \mathfrak{R}\left\{X_{1}\right\}}{\sigma^{2} \mu_{15}^{2}+\lambda_{15}^{2}}}{.}
$$

Because the surge and heave foundation forces have different phases, the maximum magnitude of the reaction force cannot be obtained from the the magnitude of the separate maximums. The maximum foundation force and time of occurrence were derived in [4] for regular waves; however, the derivation cannot be extended to irregular waves.

\section{Irregular Wave Analysis}

\subsection{Sea State Characterization}

The water surfaces in the open ocean and exposed to variable winds are typically very irregular; however, they can be seen as a superposition of multiple regular harmonic wave components. This assumption allows engineers to predict very complex irregular wave behavior in terms of theory proposed by regular waves. This superposition principle was first introduced in hydrodynamics in [20], allowing an irregular wave surface to be written as:

$$
\eta(x, t)=\sum_{n=1}^{N} A_{n} \cos \left(\sigma_{n} t-k_{n} x+\varepsilon_{n}\right),
$$

where $\varepsilon$ is a random phase angle between 0 and $2 \pi$ and $N$ denotes the number of regular wave components used to describe the sea state.

The spectral response of the WEC, $S_{R}^{+}(\sigma)$, is a function of the wave input spectrum, $S^{+}(\sigma)$. The wave spectrum is defined by:

$$
S^{+}(\sigma) d \sigma=\frac{1}{2} A(\sigma)^{2} .
$$

Each sea state may be represented as a stochastic ergodic process and the surface elevation as a stochastic input. The squared variance, $c^{2}$, of the surface elevation is equal to the area underneath the wave spectrum:

$$
c_{\eta}^{2}=\int_{0}^{\infty} S^{+} d \sigma
$$

This analysis assumes the wave elevation follows a Gaussian distribution, is stationary, and narrow-banded. Under these assumptions the peak wave amplitudes may be considered to follow a Rayleigh distribution, which is described by:

$$
P_{\eta}(\eta)=\frac{\eta}{c_{\eta}^{2}} \exp \left(\frac{-\eta^{2}}{2 c_{\eta}^{2}}\right)
$$


Relationships with statistics can be obtained by computing the spectral moments, $m_{n}$, of the wave spectrum:

$$
m_{n}=\int_{0}^{\infty} \sigma^{n} S^{+} d \sigma
$$

The spectral moments can be used to calculate the following quantities of the irregular wave surface elevation:

$$
\begin{gathered}
A_{s}=2 \sqrt{m_{0}}=\frac{8}{5} A_{\text {ave }}, \\
\sigma_{-1}=\frac{m_{0}}{m_{-1}}, \sigma_{1}=\frac{m_{1}}{m_{0}}, \sigma_{2}=\sqrt{\frac{m_{2}}{m_{0}}}
\end{gathered}
$$

where $A_{s}$ is the significant wave amplitude, $A_{\text {ave }}$ is the average wave amplitude, $\sigma_{-1}$ is the mean energy angular frequency, $\sigma_{1}$ is the mean angular frequency, and $\sigma_{2}$ is the mean zero-crossing angular frequency. The time-averaged power available for a given sea state is calculated from:

$$
P_{w}=\rho g \int_{0}^{\infty} V_{g} S^{+} d \sigma
$$

which under the deep water approximation can be shown to equal:

$$
P_{w}=\frac{\rho g^{2}}{64} H_{s}^{2} T_{-1}
$$

where $H_{S}\left(=2 A_{s}\right)$ is the significant wave height and $T_{-1}$ is the mean energy period.

\subsection{Pitch Motion in Irregular Waves}

The pitch response amplitude spectrum is given by:

$$
S_{R}^{+}=\left|\frac{\xi_{5}}{A}\right|^{2} S^{+}
$$

Each degree of freedom of the system can be considered as a random variable of zero mean, which is related to the surface elevation by means of a transfer function. The variance of pitch motion can be calculated from:

$$
c_{5}^{2}=\int_{0}^{\infty} S_{R}^{+} d \sigma
$$

The transfer function for pitch velocity is given by:

$$
G_{5}=\frac{i \sigma \xi_{5}}{A}=\frac{X_{5}}{\left[\frac{C_{55}}{i \sigma}+i \sigma\left(I_{55}+\mu_{55}\right)\right]+\left[\lambda_{55}+B_{g}\right]} .
$$

The time-averaged power can then be obtained from the squared variance of the pitch velocity:

$$
\begin{gathered}
\dot{c}_{5}^{2}=\int_{0}^{\infty}\left|G_{5}\right|^{2} S^{+} d \sigma \\
P_{T A P}=B_{g} \dot{c}_{5}^{2}
\end{gathered}
$$

where the PTO damping is assumed to be constant over all frequencies. 


\subsubsection{Foundation Reaction Forces in Irregular Waves}

The surge and heave reaction force amplitude spectrums are given by:

$$
S_{r 1}^{+}=\left|X_{r 1}\right|^{2} S^{+}, S_{r 3}^{+}=\left|X_{r 3}\right|^{2} S^{+}=\left|X_{3}\right|^{2} S^{+} .
$$

The variance of the surge and heave foundations forces are calculated from:

$$
c_{r 1}^{2}=\int_{0}^{\infty} S_{r 1}^{+} d \sigma, c_{r 3}^{2}=\int_{0}^{\infty} S_{r 3}^{+} d \sigma .
$$

The variance of the heave foundation force will not be affected by pitch motion and can be computed before optimizing the PTO damping. In addition, the variance will scale linearly with the significant wave height because of the choice spectrum that will be described in Section 5. The statistics for the heave foundation force and the corresponding zero-crossing period can be found in Appendix A.

\subsection{Optimal Constant Power-Take-Off Damping}

As known from regular wave analysis the optimal PTO damping coefficient will vary with wave frequency; however, as indicated by Eqn. (12), the incoming waves are composed of a superposition of multiple wave frequencies, and the PTO damping must be optimized for maximum power absorption. To maximize power capture, the following optimization problem was constructed. The objective function to be maximized was the time-averaged absorbed power:

$$
\max _{B_{g}^{*}} B_{g} \dot{c}_{5}^{2} .
$$

An inequality constraint was set to limit the average pitch amplitude:

$$
\frac{5}{4} c_{5} \leq \zeta_{5 \mid \max } .
$$

This formulation does not prevent the pitch amplitude from exceeding the motion constraint, but provides an initial limit given no specific wave elevation time history is used. Time-domain control methods will need to be implemented in order to make the inequality a hard constraint.

Equality constraints were used to satisfy the equation of motion:

$$
\begin{aligned}
& \text { for } j=1,2, \ldots, N \\
& \left|\xi_{5}\left(\sigma_{j}\right)\right|=\frac{A_{j}\left(\sigma_{j}\right)\left|X_{5}\left(\sigma_{j}\right)\right|}{\sqrt{\left[C_{55}-\sigma_{j}^{2}\left(I_{55}+\mu_{55}\left(\sigma_{j}\right)\right)\right]^{2}+\sigma_{j}^{2}\left[\lambda_{55}\left(\sigma_{j}\right)+B_{g}\right]^{2}}},
\end{aligned}
$$

where $N$ is the number of discretized frequencies of the wave spectrum.

\subsection{Optimal Power-Take-Off Damping Including Nonlinear Viscous Drag}

It is well known that the power capture of a device can be significantly reduced as a result of the presence of a nonlinear viscous drag term. Under regular wave excitation it is possible to apply the Lorentz linearization for continued use of spectral techniques; however, this is not possible in irregular waves. In order to incorporate a quadratic damping term the methodology proposed in [21] was used and will be briefly reviewed here. In [21] the body velocity is represented as a sum of sinusoidal frequency components:

$$
\dot{\zeta}_{5 j}=\sigma_{j}\left|\xi_{5}\right|_{j} \cos \left(\sigma_{j} t+\gamma_{j}\right)
$$

where $\gamma$ is the velocity phase obtained by taking the argument of Eqn. (23). A quasi-linear viscous damping coefficient was derived and is given by:

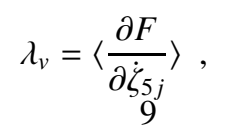


where $F$ is a general nonlinear moment and $\langle\cdot\rangle$ is the expected value of the enclosed function. The coefficient is considered quasi-linear because it depends on the complete field and therefore needs to be determined iteratively. The general form of the drag moment is defined as:

$$
M_{D}=C_{D} U|U|=C_{D}\left(\dot{\zeta_{51}}+\ldots \dot{\zeta_{5 j}}+\ldots \dot{\zeta_{5 N}}\right) \mid \dot{\zeta_{51}}+\ldots \dot{\zeta_{5 j}}+\ldots \dot{\zeta_{5 N} \mid}
$$

where $C_{D}$ is the drag coefficient. The calculation of the drag coefficient for each geometric configuration was provided in [4] using a Morison element formulation [22]. After inserting Eqn. (33) into Eqn. (32) the quasi-linear coefficient is given by:

$$
\lambda_{v}=\left\langle 2 C_{D}|U|\right\rangle .
$$

Therefore, the quasi-linear damping coefficient is proportional to the expected value of the absolute value of velocity, which again must take into account all frequency contributions. If the velocity follows a Gaussian distribution then the absolute value of the velocity follows a half-normal distribution [23]. The expected (mean) value form the half-normal distribution is equal to:

$$
\langle|x|\rangle=c \sqrt{\frac{2}{\pi}},
$$

where $x$ is the variable of interest and $c$ is the standard deviation obtained from the Gaussian distribution. Now, Eqn. (34) can be written as:

$$
\lambda_{v}=2 C_{D} \sqrt{\frac{2}{\pi}} \dot{c}_{5} .
$$

\subsection{Quantification of Hydrodynamic Radiated and Dissipated Losses}

In addition to the PTO power consumption there are hydrodynamic losses associated with the radiated waves and viscous drag effects. The concept under development has different drag coefficient and radiation damping values for each geometric configuration and is therefore of interest to determine their percentage of the total consumed power. The time-averaged power losses will be calculated from:

$$
P_{L}=\frac{1}{T} \int_{0}^{T}[\underbrace{\lambda_{v} \dot{\zeta}_{5}^{2}\left|\dot{\zeta}_{5}\right|}_{P_{\lambda_{v}}}+\underbrace{\left(K_{r} * \dot{\zeta}_{5}\right) \dot{\zeta}_{5}}_{P_{\lambda}}] d t
$$

where $K_{r}$ is the impulse response function [24] associated with pitch motion and $*$ denotes the convolution between the surrounding terms. Decomposing the pitch motion into its frequency components, individual losses can be calculated by the following:

$$
\begin{gathered}
P_{\lambda}=\int_{0}^{\infty} \lambda_{55}\left|G_{5}\right|^{2} S^{+} d \sigma, \\
P_{\lambda_{v}}=2 \sqrt{\frac{2}{\pi}} C_{D}\left(\int_{0}^{\infty}\left|G_{5}\right|^{2} S^{+} d \sigma\right)^{3 / 2} .
\end{gathered}
$$




\subsection{Fatigue Damage Analysis}

It is important that a proper design of the PTO system be performed because of its ability to control WEC motion and absorbed power. Furthermore, if an active PTO control strategy is incorporated in the WEC design it is necessary to evaluate the fatigue damage accumulation on the PTO [25] in addition to peak loads and power capacity. In this analysis, no wave-to-wave control strategy was considered and it is of interest to calculate the fatigue damage accumulation for each geometric configuration for various sea states when focused on maximizing power production.

The structure containing the PTO will be assumed to be a circular shaft, which when placed under a torsional load will need to withstand the following shear stress:

$$
\tau_{\sigma}=\frac{\tau r}{J},
$$

where $\tau_{\sigma}$ is the shear stress, $\tau$ is the applied torque, $r$ is the distance from the central axis to the stressed surface, and $J$ is the polar moment of inertia of the cross section. For this analysis, a solid circular shaft will be considered to house a direct-drive generator. The resistive torque spectrum, because of the presence of the PTO, is the product of the PTO damping and the velocity response amplitude operator:

$$
G_{\tau}=B_{g} \frac{i \sigma \xi_{5}}{A}=\frac{B_{g} X_{5}}{\left[\frac{C_{55}}{i \sigma}+i \sigma\left(I_{55}+\mu_{55}\right)\right]+\left[\lambda_{55}+B_{g}\right]} .
$$

The above equation can be used to generate a stress transfer function:

$$
S_{\tau}^{+}=\left|\frac{r}{J} G_{\tau}\right|^{2} S^{+}=\left(\frac{r}{J}\right)^{2}\left|G_{\tau}\right|^{2} S^{+} .
$$

For this analysis, the cumulative fatigue damage will be calculated based on the Palmgren-Miner's Rule, which is given by:

$$
D=\sum_{i=1}^{B} d_{i}=\sum_{i=1}^{B} \frac{n_{i}}{N_{i}},
$$

where $D$ is the cumulative fatigue damage, $d_{i}$ is the damage inflicted by each stress range, $n_{i}$ is the number of stress cycles of a particular range, $N_{i}$ is the average number of loading cycles to failure under constant amplitude loading at that stress range, and $B$ is the number of stress range intervals considered. The relationship is given by empirically derived S-N curves:

$$
N=K S^{-m},
$$

where $K$ and $m$ are material properties whereas $S$ is the specific stress range. While assuming the above S-N curve, the short-term damage incurred in the $i$-th sea state can be calculated from:

$$
D_{i}=\frac{T_{i}}{K} \int_{0}^{\infty} s^{m} f_{c i} g_{i} d s,
$$

where $T_{i}$ is the time spent in the $i$-th sea state, $s$ is the specific value of the stress range, $f_{c i}$ is the frequency of rainflow cycles for the $i$-th sea state, and $g_{i}$ is the probability density function governing $s$ in the $i$-th sea state.

If the narrow-banded assumption is maintained the peak stresses follow a Rayleigh distribution and the rainflow cycle frequency is given by the mean zero crossing frequency of the stress response. As a result, the closed-form damage expression is calculated as:

$$
D_{i}=\frac{f_{0 i} T_{i}}{K}(2 \sqrt{2})^{m} \Gamma\left(\frac{m}{2}+1\right)\left(\sqrt{m_{0 \tau}}\right)^{m},
$$

where $f_{0 i}$ is the mean zero crossing frequency of the stress spectrum, $\Gamma$ is the complete gamma function, and $m_{0 \tau}$ is the zeroth moment of the stress spectrum as given by [26]. Equation (47) will provide a conservative estimate if the 
stress process is broad-banded. A semiempirical solution was proposed by [27] to correct the narrow-banded fatigue damage calculation. The results from Eqn. (47) will be multiplied by the following scalar value:

$$
\begin{aligned}
& \kappa_{R F C}(m)=a(m)+[1-a(m)](1-\epsilon)^{b(m)}, a(m)=0.926-0.033 m, b(m)=1.587 m-2.323, \\
& \epsilon=\sqrt{1-\frac{m_{2 \tau}^{2}}{m_{0 \tau} m_{4 \tau}}},
\end{aligned}
$$

where $\epsilon$ is the bandwidth parameter, which is equal to 1 for a broad-banded process and zero for a narrow-banded process. If a value of $m=3$ is used there will be a maximum reduction of $20 \%$ in fatigue damage for a broad-banded process.

At this point in the concept development process calculating the expected lifetime of the device is not a priority. It is of greater interest to see how control of the PTO damping and flap configuration alters the results of Eqn. (47) for a given sea state. For reporting purposes, the following damage value will be used:

$$
D_{i}^{*}=\frac{K}{T_{i}}\left(\frac{\left(\frac{J}{R}\right)^{2 m}}{(2 \sqrt{2})^{m} \Gamma\left(\frac{m}{2}+1\right)}\right) D_{i}=f_{0 i}\left(\sqrt{m_{0 \tau}}\right)^{m} .
$$

Thus, the only material coefficient required is the exponent $m$, which after a review of the S-N curves in [28] provides a value of three for steel in seawater with cathodic protection.

As seen in Eqn. (50), the dominant contribution will be a result of the exponent of the expected torque amplitude. A doubling in the PTO torque leads to an eight times increase in damage. Therefore, load shedding in greater sea states must be considered to extend the lifetime of the PTO structure. The fatigue damage is also proportional to the zero-crossing frequency; however, this will not deviate significantly from the resonance frequency of each geometric configuration. At most the ratio between the zero-crossing frequency between the one- and four-flap geometries would be a factor of two, which is quickly dwarfed by the cube of the PTO torque amplitude.

\section{Results from Irregular Wave Optimization}

To complete the analysis presented for this work an irregular wave spectrum needed to be selected. The choice of spectrum can have a significant effect on the expected power performance of the WEC [29], because it is especially sensitive to the narrowbandedness of the sea state [30]. For this analysis, the Bretschneider spectrum [31] was chosen and is given by:

$$
S^{+}=\frac{5}{16} \frac{\sigma_{p}^{4}}{\sigma^{5}} H_{s}^{2} \exp \left[-\frac{5}{4}\left(\frac{\sigma_{p}}{\sigma}\right)^{4}\right],
$$

where $\sigma_{p}$ is the modal (peak) angular frequency of the wave spectrum and $H_{s}$ is the significant wave height traditionally defined as the mean wave height of the highest third of the waves. The spectral moments can be calculated numerically from the available frequency data; however, the definition of the Bretschneider spectrum allows for derivation of the following analytic expressions:

$$
\left.\begin{array}{c}
m_{0}=\left(\frac{H_{s}}{4}\right)^{2} \\
m_{2}=\frac{\sqrt{5 \pi}}{2} \sigma_{p}^{2} m_{0}
\end{array}\right\} \Rightarrow \sigma_{2}=\sqrt{\frac{m_{2}}{m_{0}}}=\left(\frac{5 \pi}{4}\right)^{1 / 4} \sigma_{p} .
$$

The fourth spectral moment diverges slowly as $\sigma \rightarrow \infty$; thus, an analytic solution to the bandwidth of the sea state is not possible. Though an approximation was derived for the fourth spectral moment [32] leading to:

$$
\begin{gathered}
m_{4} \approx 7.049 m_{0} \sigma_{p}^{4} \text { for } \sigma<5 \sigma_{p}, \\
\therefore \epsilon \approx 0.665,
\end{gathered}
$$

where the bandwidth of the wave spectrum is constant regardless of the peak period and significant wave height. 


\subsection{Linear Analysis}

Short-term statistics were calculated for four flap configurations in irregular seas with a peak period and significant wave height that spanned the range of 5-20 s and 1-4 m respectively. The average pitch amplitude of motion was limited to 30 degrees to adhere to the requirements for applying linear hydrodynamic theory and constraints associated with maximum PTO stroke length. In this analysis the rate of change in geometry is considered to be slow such that the geometry would transition over several minutes based on measured or predicted changes in the spectrum peak period and significant wave height. The linear PTO damping coefficient was unconstrained to obtain the upper limit on power production. It was shown in [4] that limits on the linear PTO damping coefficient can reduce device performance, most significantly off resonance and result in conditions where motion constraints could be exceeded.

The short-term power performance statistics from the linear inviscid analysis are plotted in Fig. 5. At the smallest significant wave heights the absorbed power is maximized at the resonance frequency for each geometric configuration providing the greatest capture widths. In these sea states the device oscillates almost freely with little influence from the pitch amplitude constraint, and the most efficient energy harvesting is expected to occur near the resonance frequency [19]. This also corresponds to the conditions in which the radiated power comprises the largest percentage of the sum of the absorbed and radiated power; however, as the significant wave height is increased the largest drop in capture width occurs near the resonant frequency and the maximum power absorption is observed at frequencies higher than resonance. This is a consequence of the motion constraint placed on the pitch amplitude of motion. For a strongly constrained system the results from [33] can be used to show that the maximum absorbed power, under regular wave excitation, is given by:

$$
\frac{P_{\max }^{c}}{A^{2}}=\frac{1}{2} \sigma\left|X_{5}\right| \frac{\zeta_{5 \mid \max }}{A} .
$$

Under these conditions the maximum power follows the wave-exciting force magnitude, rather than resonant motion, which for all geometries is largest at approximately $0.16 \mathrm{~Hz}$, which was observed in Fig. 3b. This affects the one-flap configuration the least, because its resonant frequency is the lowest of all the geometries and other than for results at the end of the low-frequency regime the nondimensional capture width has the least variation with an average value of 0.2 . The one-flap geometry produces the greatest time-averaged power for most moderate-to-large sea states; whereas the four-flap geometry never provides the maximum achievable power despite being a resonant device. Thus, if developers are concerned only with optimization of the absorbed power with an unconstrained PTO, the design should converge toward the zero-flap configuration, which most closely resembles a conventional OSWEC. Yet, it is necessary to weight the absorbed power by the corresponding forces on both the PTO and foundation before load shedding geometric configurations can be neglected.

The corresponding short-term load statistics are plotted in Fig. 6. The optimum PTO damping coefficient increases as each additional flap is closed which follows the increase in wave radiation damping. Under low-to-moderate sea states the largest PTO damping coefficients occur away from the resonance frequency because an increase in PTO torque is needed to offset the drop in pitch velocity. As the significant wave height is increased the damping coefficient increases near resonance to meet the pitch amplitude constraint. The ratio between the maximum PTO damping and torque between the one- and four-flap geometries is approximately 20 and 6, respectively; however, the ratio between the maximum absorbed power is only 2.2. Therefore, the loading on the PTO structure increases faster than the absorbed power as each additional flap is closed. As a result, the fatigue damage accumulation between the oneand four-flap configuration is two orders of magnitude larger. The bandwidth of the torque spectrum is minimized near the resonance frequency where the narrowbandedness assumption is strongest; however, as the significant wave amplitude is increased the bandwidth increases, because the energy normally dominated by the oscillating modes near resonance is lost due to the presence of the motion constraint. The standard deviation in the surge foundation force is generally a maximum near resonance, but as the significant wave height is increased the peak is reduced and becomes roughly constant across all wave spectrum peak frequencies. Furthermore, in the largest sea states the ratio of the surge foundation force between the one- and four-flap geometries is approximately 2.8 , which highlights the ability of the proposed WEC to continue operation in more severe sea states while producing a baseline power level. In order to optimize the absorbed power while minimizing loads the proposed WEC design would operate in the one-flap geometry for low-to-moderate sea states. As the waves grow from moderate-to-severe sea states additional flaps will be opened to meet the safety factors set for load on the PTO and foundation. The absorbed power may not increase 

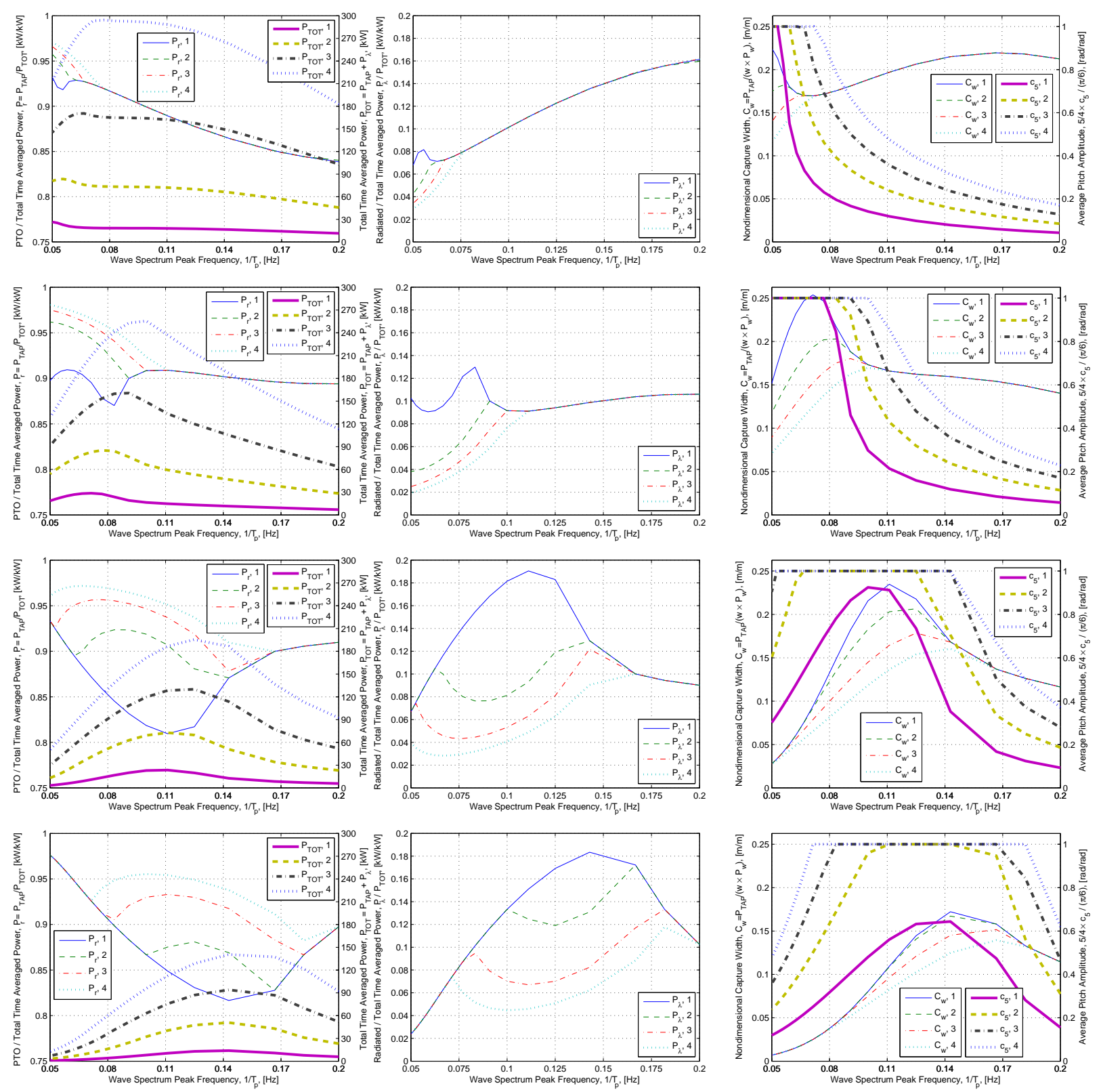

Figure 5. The linear power performance parameters for each geometric configuration with the following PTO damping and pitch motion amplitude constraints: 20 MN.m.s and $\pi / 6$. The left, middle, and right columns correspond to the following values: left: ratio of PTO time absorbed power to the total consumed power and the total consumed power, middle: ratio of radiated losses to total consumed power, right: nondimensional capture width and average pitch-displacement amplitude. Each plot shows the variation in performance for different significant wave heights of $1,2,3$, and $4 \mathrm{~m}$. Each row corresponds to a different geometry beginning with 1 open flap and the following rows depicts 2, 3, and 4 open flaps. 
with wave height in larger sea states because of the reduction of loads when each additional flap is opened, but due to the increase in wave height a large baseline power level can be maintained.

\subsection{Nonlinear Analysis}

The short-term power performance statistics from the nonlinear viscous analysis are plotted in Fig. 7. The inclusion of the quadratic viscous damping moment leads to a significant decrease in the time-averaged absorbed power for each geometric configuration. The average reduction in absorbed power between the linear and nonlinear cases is $30 \%, 40 \%, 34 \%$, and $22 \%$ for the one-, two-, three-, and four-flap configurations, respectively. Furthermore, the average pitch motion amplitude never reaches the imposed limit; thus, results will not change when softening the motion constraint. The power lost as a result of the viscous drag moment is $20 \%-30 \%$ of the sum of absorbed, radiated, and dissipated power in the higher sea states. The percentage of the radiated power follows a nearly linear trend with the wave spectrum peak frequency and decreases with each additional open flap, which is coincident with the reduction in wave damping coefficient. The capture width remains greatest at the lowest sea states, because the amplitude of motion is low enough that the effect from the nonlinear drag moment is minimized; however, the local resonance peaks are lost and performance is less variable with peak frequency. Furthermore, there is distinct step reduction in capture width as each additional flap is opened, and unlike the linear analysis the one-flap geometry has the greatest nondimensional capture width for all sea states with an upper limit of 0.2.

The corresponding short-term load statistics from adding the nonlinear viscous drag moment are plotted in Fig. 8. The optimum PTO damping coefficient continues to increase as each additional flap is closed and with significant wave height; however, the variance in PTO torque, per significant wave height, remains nearly constant under all sea states because of the reduction in pitch motion per significant wave height. The average ratio between the maximum PTO damping and torque between the one- and four-flap geometries has slightly increased to 21 and 7, respectively, whereas the ratio between the maximum PTO absorbed power is approximately 2.4. The fatigue damage accumulation rate between the one- and four-flap configuration remains roughly two orders of magnitude larger; however, the local peak in fatigue rate that was present near the resonant frequency of each geometry in the linear analysis has also been eliminated. The highest fatigue damage accumulation rate now occurs in the high-frequency regime where the PTO torque amplitude and zero-crossing frequency is greatest. The bandwidth of the torque spectrum is still minimized near the resonance frequency, though it is significantly noticeable for the three- and four-flap configurations only. In addition, the bandwidth parameter has increased for all geometric configurations, which indicates that the viscous drag moment is spreading energy among a wider range of oscillating modes. The standard deviation in the surge foundation force continues to be a maximum near resonance, but shows a significant reduction in variation with significant wave height. The ratio of the surge foundation force between the one- and four-flap geometries is approximately 3.4 , a considerable increase over the linear analysis. This is a result of the $12 \%$ average increase in the surge foundation force for the one-flap configuration compared to the $9 \%$ reduction for the four-flap configuration when including the viscous drag moment. Inclusion of the viscous drag moment results in both a reduction in the pitch motion amplitude and a shift in phase. As denoted in Eqn. (11), in order to eliminate the surge foundation force the OSWEC must oscillate at a specific amplitude and phase. Therefore, many factors can contribute to either an increase or decrease in surge foundation force, which is dependent on the magnitude of the chosen drag coefficient and its contribution to the body dynamics.

\section{Conclusion}

This research has used spectral techniques to ascertain the irregular wave performance of a novel WEC concept that combines an OSWEC with active control surfaces. The variable geometry provides an additional degree of freedom that can be used to alter the hydrodynamic properties that can, on top of the PTO, be used for greater control of device performance. The spectral analysis was first completed using linear hydrodynamic theory in which an optimization problem was constructed using an objective function based on the time-averaged absorbed power. The results showed the WEC design was effective at tuning the hydrodynamic characteristics such that the resonant frequency of the device matched the dominant wave-excitation frequency leading to the optimum conditions for power extraction in low-tomoderate sea states. Furthermore, as the sea states transitioned from moderate-to-severe, the design was shown to be capable of reducing both foundation and PTO loads while increasing the capacity factor and maintaining a baseline 

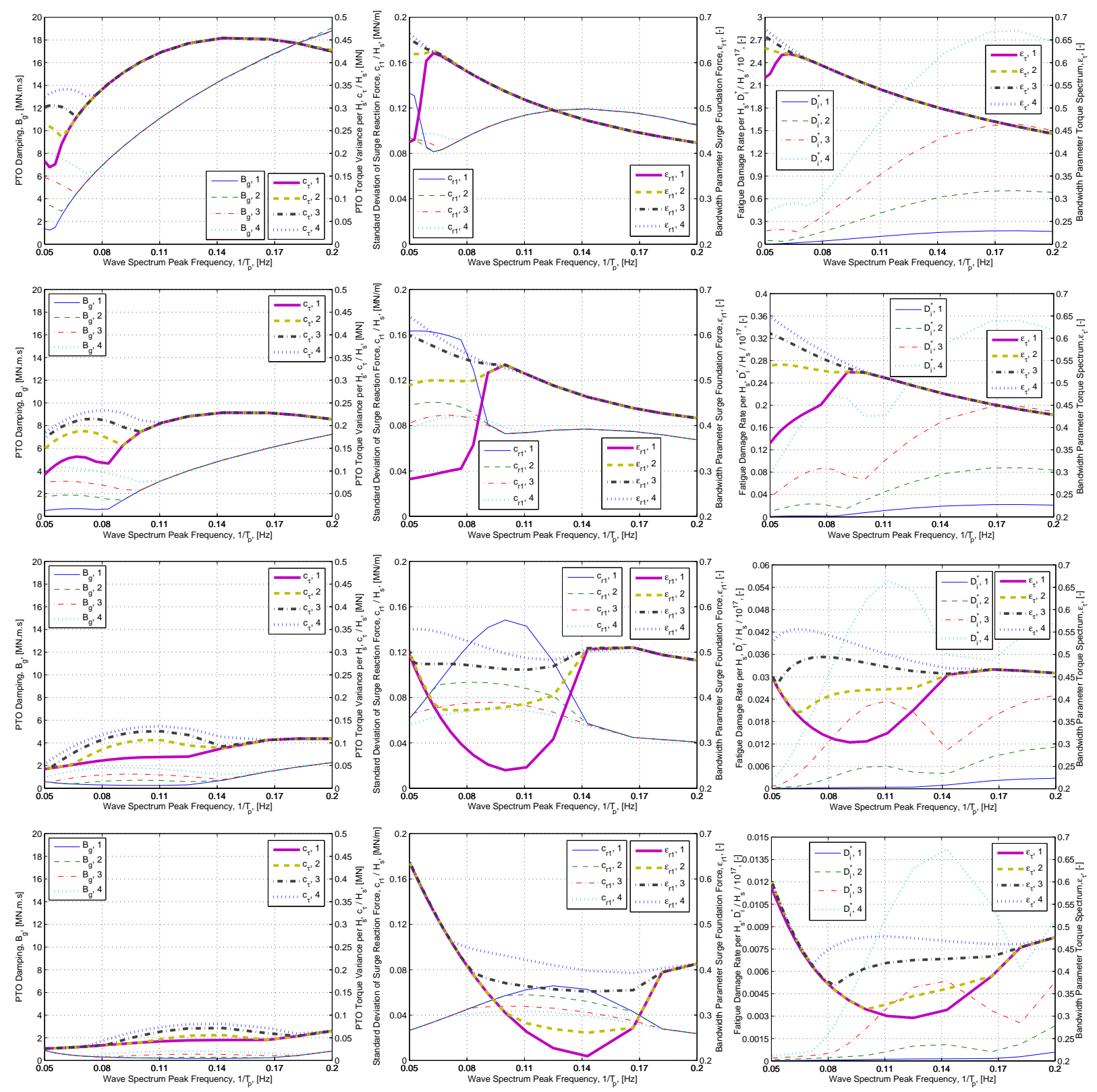

Figure 6. The linear load performance analysis for each geometric configuration with the following PTO damping and pitch motion amplitude constraints: 20 MN.m.s and $\pi / 6$. The left, middle, and right columns correspond to the following values: left: PTO damping and torque, middle: standard deviation of surge reaction force and surge foundation force spectrum bandwidth parameter, right: fatigue damage rate and torque spectrum bandwidth parameter. Each plot shows the variation in performance for different significant wave heights of 1, 2, 3, and $4 \mathrm{~m}$. Each row corresponds to a different geometric configuration beginning with 1 open flap and the following rows depict 2,3 , and 4 open flaps. 

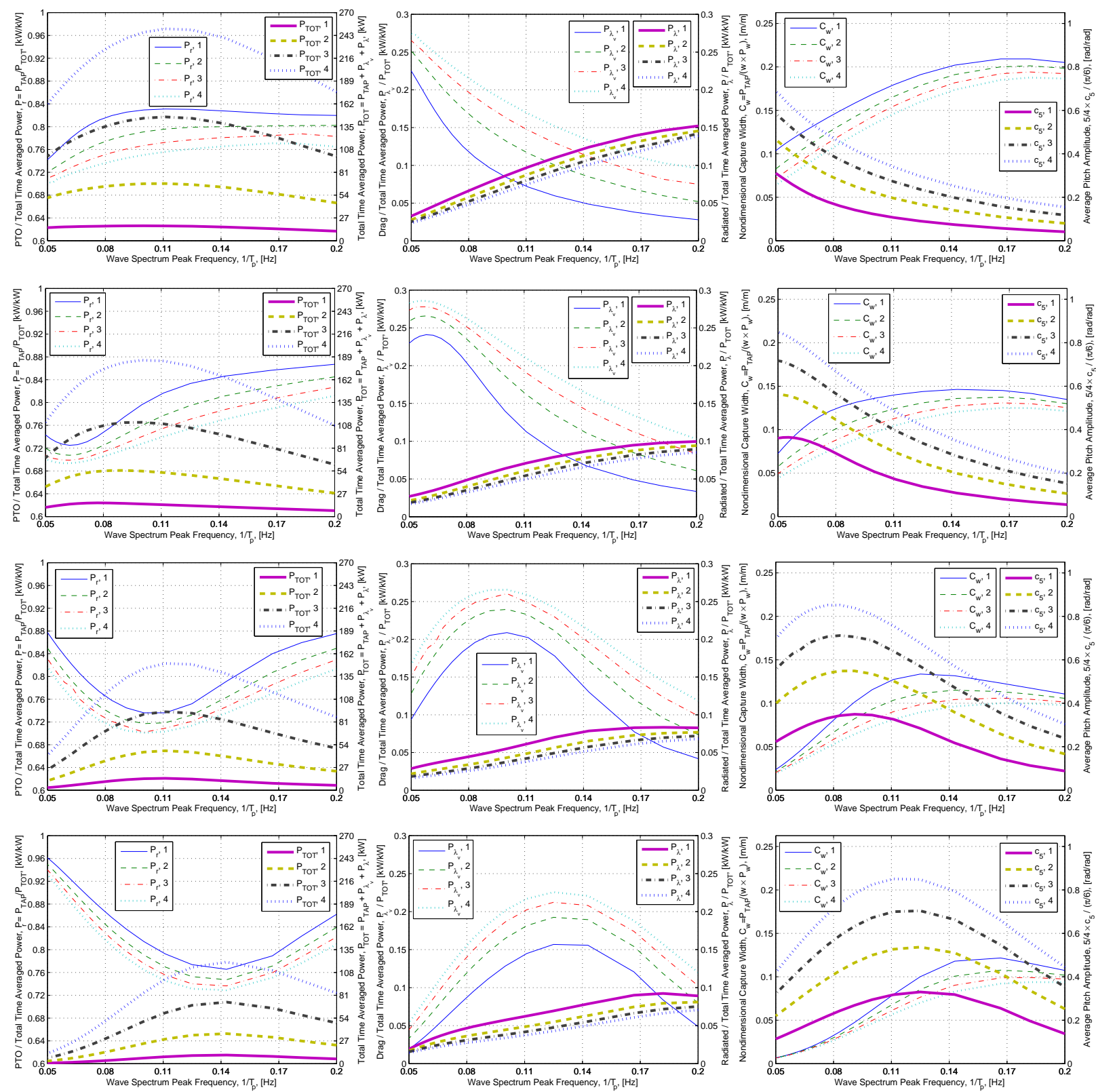

Figure 7. The nonlinear power performance parameters for each geometric configuration with the following PTO damping and pitch motion amplitude constraints: 20 MN.m.s and $\pi / 6$. The left, middle, and right columns correspond to the following values: left: ratio of PTO time absorbed power to the total consumed power and the total consumed power, middle: ratio of viscous drag and radiated losses to total consumed power, right: nondimensional capture width and average pitch-displacement amplitude. Each plot shows the variation in performance for different significant wave heights of $1,2,3$, and $4 \mathrm{~m}$. Each row corresponds to a different geometric configuration beginning with 1 open flap and the following rows depict 2,3 , and 4 open flaps. 

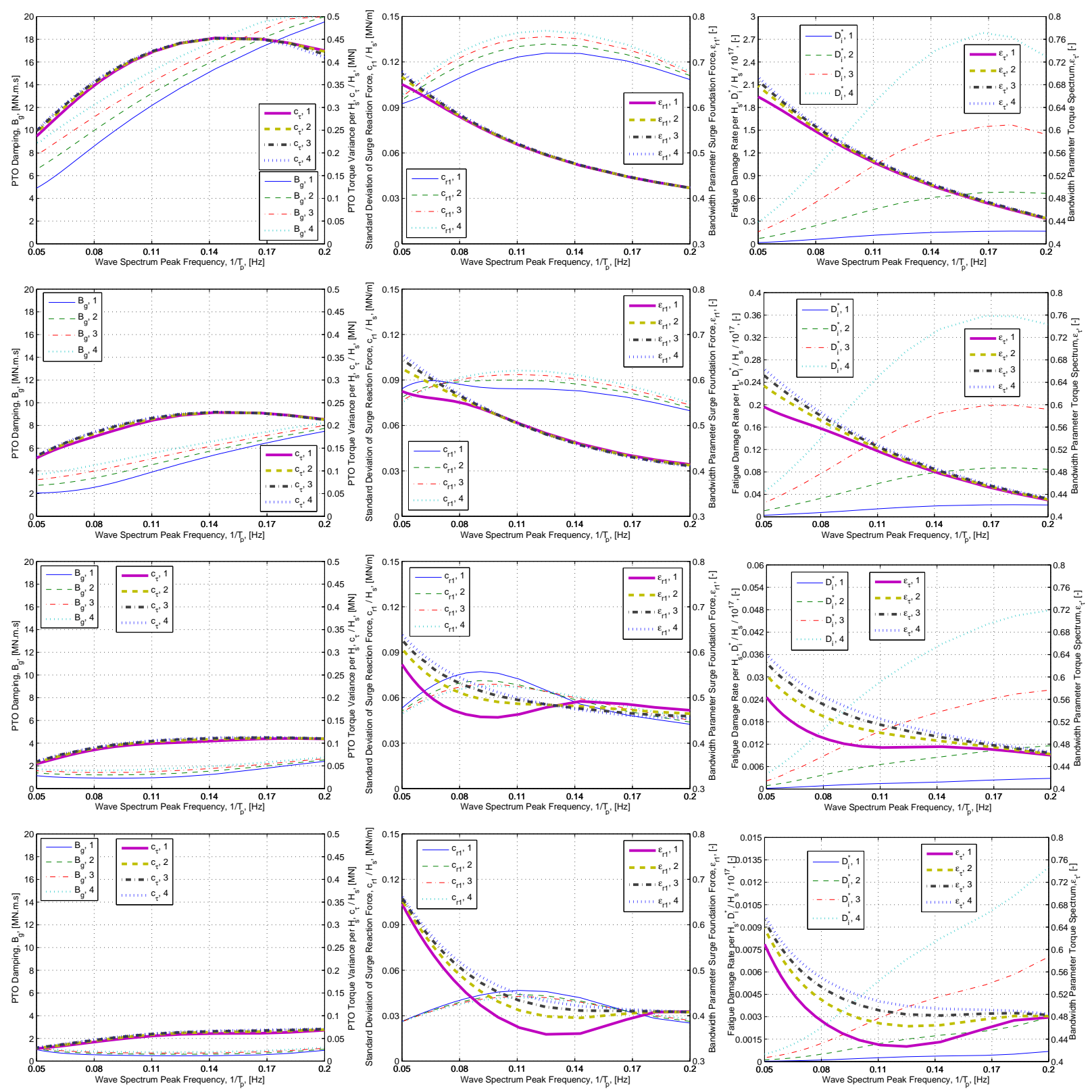

Figure 8. The nonlinear loads analysis for each geometric configuration with the following PTO damping and pitch motion amplitude constraints: 20 MN.m.s and $\pi / 6$. The left, middle, and right columns correspond to the following values: left: PTO damping and torque, middle: standard deviation of surge reaction force and surge foundation force spectrum bandwidth parameter, right: fatigue damage rate and torque spectrum bandwidth parameter. Each plot shows the variation in performance for significant wave heights of 1, 2, 3, and $4 \mathrm{~m}$. Each row corresponds to a different geometric configuration beginning with 1 open flap and the following rows depict 2,3 , and 4 open flaps. 
power output. This work has attempted to highlight the issues that arise when solely maximizing power absorption for a given sea state, because it generally corresponds to greater loads requiring increased structural mass and a higher levelized cost of energy.

The hydrodynamic modeling was further improved by introducing a nonlinear quadratic viscous drag moment. A quasi-linear viscous damping coefficient was derived that was dependent on the complete field of motion, forcing the optimization problem to obtain the solution iteratively. Results showed that the constraint on the pitch amplitude of motion was never encountered even in the largest sea states, thereby reducing the need for large end stop moments. As a result, there was an average reduction of $30 \%$ in time-averaged absorbed power and a flattening of the capture width plots. Furthermore, unlike in the linear analysis the one-flap geometry provides the best power performance with an almost step reduction as each additional flap is opened. Despite the reduction in pitch motion, the ratio of the foundation and PTO loads between the one- and four-flap geometries remained approximately constant. Additionally, maximum fatigue damage accumulation rate has not changed significantly and remains approximately two orders of magnitude higher between the same two geometries, though the local peaks about the resonance frequencies have been eliminated. The largest effects of the quadratic viscous drag moment have been flattening the performance curves and reducing the sensitivity with respect to the significant wave amplitude.

The spectral techniques described in this article can be completed significantly faster than running time-domain simulations; however, results can only be reported in terms of motion and power statistics. This is a limitation in terms of evaluating the performance of the device under strict amplitude constraints, which will require a real-time controller with an adequate prediction of the future incoming wave elevation. This is caused by the loss of phase information when using the spectral representation of the sea state, which makes it difficult to calculate quantities such as the magnitude of the foundation reaction force. The PTO was assumed to provide a constant linear damping coefficient for a given sea state; however, wave-to-wave control of the PTO system can be used to further optimize power absorption and assist in load shedding. Despite these limitations the techniques presented in this work provide a quick and efficient methodology for evaluating the irregular wave performance of a one-degree-of-freedom WEC at a midfidelity level. The results of this research have shown the ability of the proposed WEC concept to adapt to various wave climates while continuing to optimize power, increase capacity factor, and reduce structural loads.

\section{Acknowledgments}

This work was supported by the U.S. Department of Energy under Contract No. DE-AC36-08GO28308 with the National Renewable Energy Laboratory. Funding for the work was provided by the DOE Office of Energy Efficiency and Renewable Energy, Wind and Water Power Technologies Office. 


\section{References}

[1] Weber J, Costello R, Ringwood J. WEC technology performance levels (TPLs) - Metric for successful development of economic wec technology. In: Proc. of the 10th european wave and tidal energy conference; 2013.

[2] Yu YH, Jenne DS, Thresher R. Reference model 5 (RM5): Oscillating surge wave energy converter. National Renewable Energy Laboratory; 2015. Technical report NREL/TP-5000-62861.

[3] Musial W, Lawson MJ, Rooney S. Marine hydrokinetic technology (MHK) instrumentation, measurement, and computer modeling workshop. National Renewable Energy Laboratory; 2013. Technical report NREL/TP-5000-57605.

[4] Tom N, Lawson MJ, Yu YH, Wright AD. Parameter study for a wave energy converter with active control surfaces. Renewable Energy 2016; (under review).

[5] Siegel SG, Fagley C, Romer M, McLaughlin TE. Experimental wave cancellation using a cycloidal wave energy converter. In: Proc. of the 9th european wave and tidal energy conference; 2011.

[6] Pecher A, Kofoed JP, Larsen T, Marchalot T. Experimental study of the Weptos wave energy converter. In: Proc. of the 31st International Conference on Ocean, Offshore, and Arctic Engineering; 2012. OMAE2012-83751.

[7] Kurniawan A, Moan T. Characteristics of a pitching wave absorber with rotatable flap. Energy Procedia 2012;20:134-147.

[8] Whittaker TW, Folley M. Nearshore oscillating wave surge converters and the development of Oyster. Philos. Trans. R. Soc. A 2012;370:345-364.

[9] Lucas J, Livingstone M, Vuorinen M, Cruz J. Development of a wave energy converter (WEC) design tool - application to the WaveRoller WEC including validation of numerical estimates. In: Proc. of the 4th International Conference on Ocean Energy; 2012.

[10] Ramudu E. Ocean wave energy-driven desalination systems for off-grid coastal communities in developing countries. In: Proc. of the IEEE Global Humanitarian Technology Conference; 2011. pp. 1129-1142.

[11] Pecher J, Kofoed J, Espedal J, Hagberg S. Results of an experimental study of the Langlee wave energy converter. In: Proc. of the 20th International Offshore and Polar Engineering; 2010. pp. 877-885.

[12] Gomes RPF, Lopes MFP, Henriques JCC, Gato LMC, Falcão AFO. The dynamics and power extraction of bottom-hinged plate wave energy converters in regular and irregular waves. Ocean Engineering 2015;96:86-99.

[13] Flocard F, Finnigan TD. Increasing power capture of a wave energy device by inertia adjustment. Applied Ocean Research 2012;34:126-134.

[14] Falcão AF de O, Rodrigues RJA. Stochastic modelling of OWC wave power plant performance. Applied Ocean Research 2002;24:59-71.

[15] Bachynski EE, Young YL, Yeung RW. Analysis and optimization of a tethered wave energy converter in irregular waves. Renewable Energy 2012;48:133-145.

[16] Caska AJ, Finnigan TD. Hydrodynamic characteristics of a cylindrical bottom-pivoted wave energy absorber. Ocean Engineering 2008;35:6-16.

[17] Tom N, Lawson M, Yu YH, Wright AD. Preliminary analysis of an oscillating surge wave energy converter with controlled geometry. In: Proc. of the 11th european wave and tidal energy conference; 2015.

[18] WAMIT Version 7.0 User Manual, http://www.wamit.com; 2013.

[19] Evans DV. A theory for wave-power absorption by oscillating bodies. Journal of Fluid Mechanics 1976;77(1):1-25.

[20] St. Denis M, Pierson WJ. On the motions of ships in confused seas. In: Annual meeting of The Society of Naval Architects and Marine Engineers; 1953. pp. 280 - 356.

[21] Folley M, Whittaker TW. Spectral modelling of wave energy converters. Coastal Engineering 2010;57:892-897.

[22] Morison JR, Johnson JW, Schaaf SA. The force exerted by surface waves on piles. Journal of Petroleum Technology 1950;2(5):149-154.

[23] Byers RH. Half-normal distribution. In: Encyclopedia of Biostatistics 2005;4.

[24] Cummins WE. The impulse response function and ships motions. Schiffstechnik 1962;9:101-109.

[25] Zurkinden A, Lambertsen SH, Damkilde L, Gao Z, Moan T. Fatigue analysis of a wave energy converter taking into account different control strategies. In: Proc. of the 32nd International Conference on Ocean, Offshore, and Arctic Engineering; 2013. OMAE2013-10864.

[26] Det Norske Veritas. Riser Fatigue. Det Norske Veritas; 2010. Technical report DNV-RP-F204.

[27] Wirsching PH, Light MC. Fatigue under wide band random stresses. Structural Division of the American Society of Civil Engineers 1980;106(7):1593-1607.

[28] Det Norske Veritas. Fatigue design of offshore steel structures. Det Norske Veritas; 2011. Technical report DNV-RP-C203.

[29] Clabby D, Henry A, Folley M, Whittaker TW. The effect of the spectral distribution of wave energy on the performance of a bottom hinged flap type ave energy converter. In: Proc. of the 31st International Conference on Ocean, Offshore, and Arctic Engineering; 2012. OMAE201283398.

[30] Saulnier JB, Ricci P, Pontes MT, Falcão AFO. Spectral bandwidth and WEC performance assessment. In: Proc. of the 7th European wave and tidal energy conference; 2007, pp. 1 - 10.

[31] Ochi MK. Ocean Waves: The Stochastic Approach. In: Dyer I, Eatock Taylor R, Newman JN, Price WG, editors. Cambridge Ocean Technology Series, Cambridge: Cambridge University Press; 2005, pp. 34 - 40.

[32] Beck RF, Cummins WE, Dalzell JF, Mandel P, Webster WC. Motions in Waves and Controllability. In: Lewis EV, editor. Principles of Naval Architecture, New Jersey: Society of Naval Architects and Marine Engineers; 1989.

[33] Evans DV. Maximum wave-power absorption under motion constraints. Applied Ocean Research 1981;3(4):200-203. 
Figure A.9. The standard deviation in heave reaction force and the associated bandwidth parameter for each flap configuration. The numbers in the legend correspond to configurations with $1,2,3$, and 4 open flaps starting from the top.

\section{Appendix A. Heave Foundation Reaction Force Statistics}

As discussed in Section 3.3, the heave reaction force is independent of pitch motion and controllable only through the device geometry. When the flaps are closed a small surface area normal to the heave direction corresponds to a lower dynamic heave force as shown in Fig. A.9. The largest increase in the heave reaction force occurs between the one- and two-open flap configurations, which is consistent with the exponential decrease in hydrodynamic pressure as one moves down the water column. The heave reaction force is greatest in the high-frequency regime as the wave conditions move more towards deep water and the water particle orbitals gain a greater vertical component. Furthermore, the difficulty in calculating the maximum magnitude of the reaction force is highlighted in Fig. A.10. The surge and heave foundation forces have different zero-crossing periods with the heave force oscillating faster than the surge force. The current spectral analysis cannot accurately estimate the variance in the magnitude of the foundation reaction force because of the different oscillation speeds requiring time-domain simulations to be completed to obtain better estimates on the maximum force and fatigue damage on the OSWEC foundation.

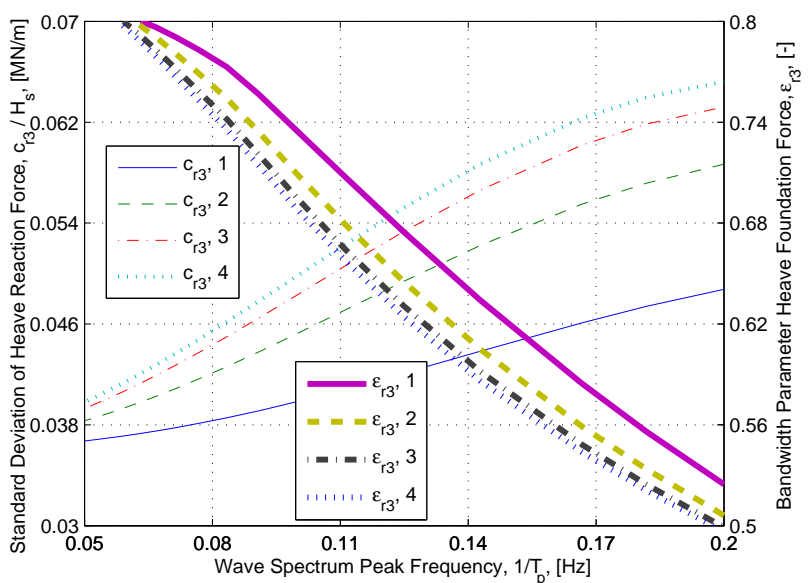




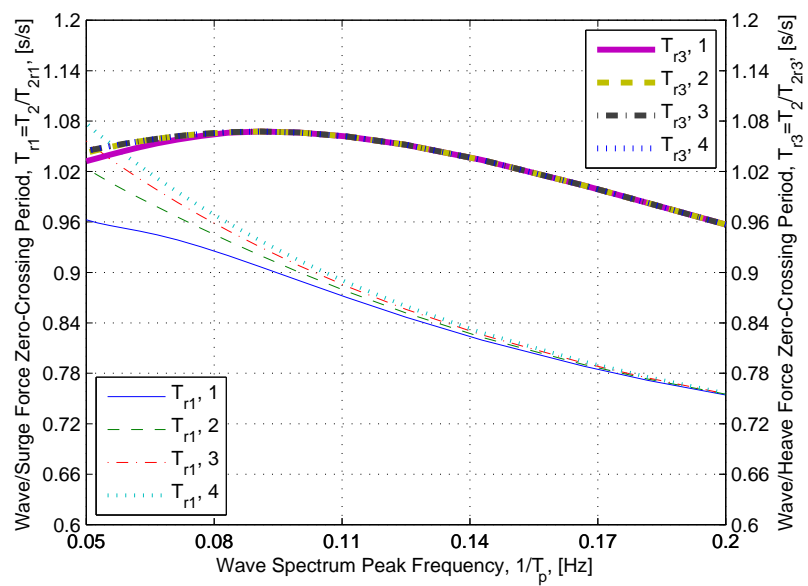

(a) One flap open.

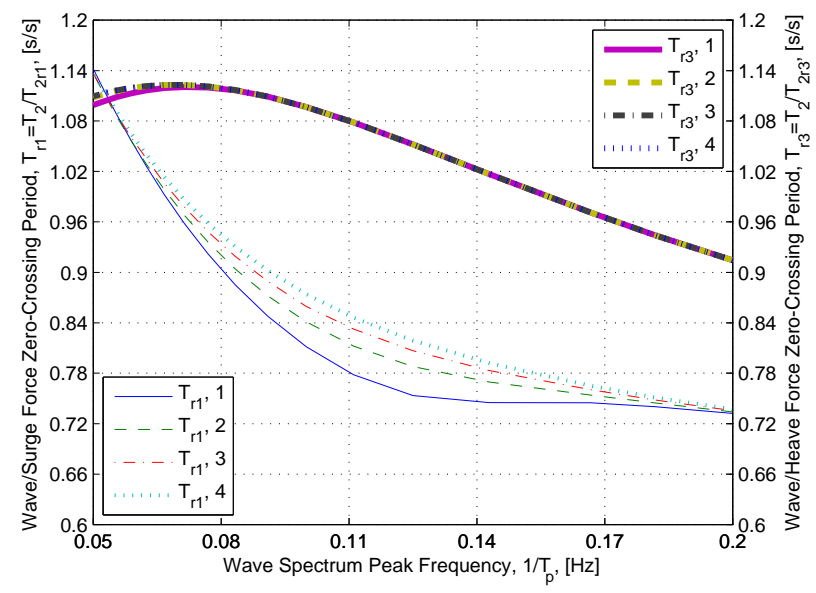

(c) Three flaps open.

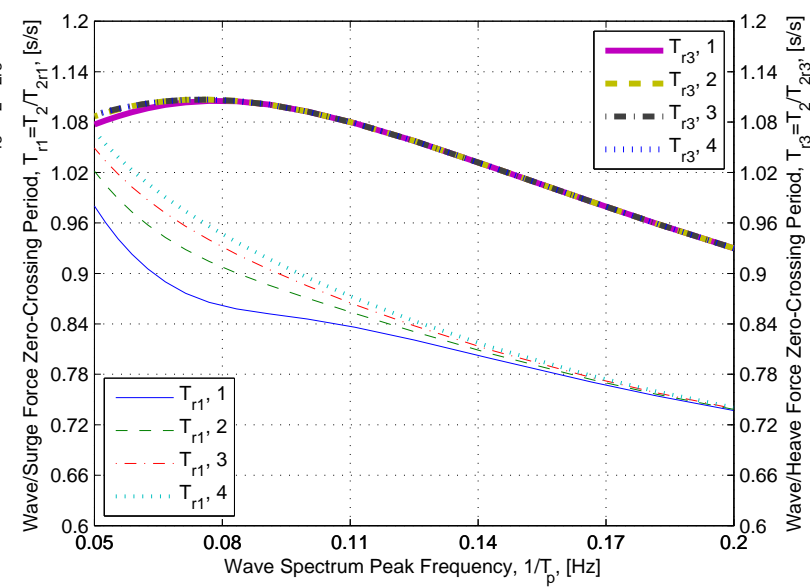

(b) Two flaps open.

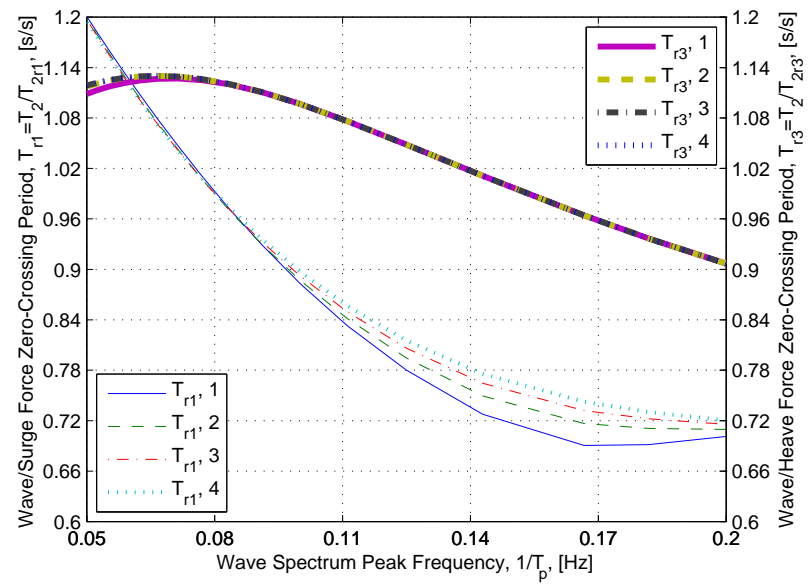

(d) Four flaps open.

Figure A.10. Ratio of zero-crossing period between the wave spectrum and the surge and heave foundation forces for each geometric configuration. 